\title{
ACUERDOS PRODUCTIVOS DESDE LA VISIÓN DEL DESARROLLO LOCAL: EL ROL DE LA INNOVACIÓN*
}

\author{
María Verónica Alderete ${ }^{* *}$ \\ Recibido: mayo 09 de 2012 • Aceptado: mayo 24 de 2013
}

\section{RESUMEN}

Este trabajo realiza una discusión teórica en torno a la importancia de las aglomeraciones productivas desde el punto de vista del desarrollo local, con especial énfasis en el papel que cumplen como promotores de innovación. El concepto teórico de aglomeraciones productivas ha evolucionado en función de la importancia de los actores locales para alcanzar los objetivos del desarrollo local. Tal evolución teórica tiene su impacto sobre la formulación de las políticas de desarrollo, incluidas aquellas para promover la innovación. La revisión teórica se focaliza en el desarrollo de los sistemas de producción e Innovación locales estudiados por RedeSist, Brasil. Esta perspectiva no pretende determinar normas generales de aplicación, dadas las singularidades del desarrollo regional sino contribuir a la discusión teórica y normativa para promover el desarrollo local.

\section{PALABRAS CLAVE}

Acuerdos productivos, desarrollo local, innovación, Brasil

\section{CLASIFICACIÓN JEL}

R10, R58, 031, L14

\section{CONTENIDO}

Introducción; 1. Marco teórico; 2. Antecedentes sobre aglomeraciones productivas, innovación y desarrollo territorial; 3. Estudios empíricos sobre acuerdos de producción e innovación locales, 4. Nuevas políticas de Innovación en acuerdo de producción; 5 . Consideraciones finales; Bibliografía.

\footnotetext{
Artículo de Revisión, producto del proyecto de investigación "Conductas y estrategias de pymes regionales en los mercados Interno y de Exportación" y adscrito al grupo de investigación PGI, Departamento de Economía, Universidad Nacional del Sur, Argentina. Proyecto ejecutado en el período: 2011-2012. La autora agradece al Dr. Miguel Bacic por sus aportes como director de una beca de investigación Postdoctoral de CONICET en La Universidad Estadual de Campinas, Brasil.

** Licenciada en Economía, Departamento de Economía, Universidad Nacional del Sur, Bahía Blanca, Argentina. Doctora en Economía, Departamento de Economía, Universidad Nacional del Sur, Bahía Blanca, Argentina. Investigadora Asistente del Instituto de Investigaciones Económicas y Sociales del Sur (IIESS)-CONICET, Argentina. Correo electrónico: mvalderete@iiess-conicet.gob.ar.
} 


\section{PRODUCTIVE AGREEMENTS FORM A LOCAL DEVELOPMENT SCOPE: THE ROLE OF INNOVATION \\ ABSTRACT}

This paper provides a theoretical discussion about the importance of production clusters from a local development scope, with special emphasis on the role they have as innovation promoters. The theoretical concept of productive clusters has evolved based the importance that local actors have in achieving the local development objectives. This theoretical development has an impact on the formulation of development policies, including those to promote innovation. The theoretical review focuses on local production and Innovation systems studied by RedeSist, Brazil. This perspective is not intended to determine general rules of application, given the particularities of regional development but to contribute to the theoretical and normative discussion in order to promote local development.

\section{KEY WORDS}

Productive agreements, local development, innovation, Brazil

\section{JEL CLASSIFICATION}

R10, R58, O31, L14

\section{CONTENT}

Introduction; 1. Theoretical framework; 2. Productive clusters background; 3.Empiric studies about production agreements and local innovation, 4 . New innovation policies in production agreements; 5 . Final considerations; Bibliography.

\section{ACORDOS PRODUTIVOS DESDE A VISÃO DO DESENVOLVIMENTO LOCAL: O ROL DA INNOVACIÓN \\ RESUMO}

Este trabalho realiza uma discussão teórica em volta à importância das aglomerações produtivas desde o ponto de vista do desenvolvimento local, com especial ênfase no papel que cumprem como promotores de inovação. O conceito teórico de aglomerações produtivas tem evoluído em função da importância dos atores locais para conseguirem os objetivos do desenvolvimento local. Tal evolução teórica tem seu impacto sobre a formulação das políticas de desenvolvimento, incluídas aquelas para promover a inovação. A revisão teórica se focaliza no desenvolvimento dos sistemas de produção e inovação locais estudados por RedeSist, Brasil. Esta perspectiva não pretende determinar normas gerais de aplicação, dadas as singularidades do desenvolvimento regional e sim, contribuir à discussão teórica e normativa para promover o desenvolvimento local.

\section{PALAVRAS CHAVE}

Acordos produtivos, desenvolvimento local, inovação, Brasil

\section{CLASIFICAÇÃO JEL}

R10, R58, 031, L14

\section{CONTEÚDO}

Introdução; 1. Marco teórico; 2. Antecedentes sobre aglomerações produtivas, inovação e desenvolvimento territorial; 3. Estudos empíricos sobre acordos de produção e inovação locais, 4. Novas políticas de inovação em acordo de produção; 5. Considerações finais; Bibliografia. 
Acuerdos productivos desde la visión del desarrollo local: el rol de la innovación

\section{INTRODUCCIÓN}

Durante los últimos años, gran parte de las investigaciones que estudian los cambios socioeconómicos y las consiguientes transformaciones territoriales han hecho hincapié en el fenómeno de la innovación, así como en el espacio de interacción conformado por las redes entre los actores locales. La innovación, como factor que impulsa la generación e incorporación de conocimientos, permite a los diferentes actores locales, como las empresas, insertarse de manera competitiva y propender al desarrollo de sus ámbitos territoriales.

Una revisión teórica del fenómeno de la innovación permite observar cierta diversidad de enfoques. Algunos estudios parten de la perspectiva de las firmas según la visión neo-schumpeteriana (Freeman, 1988; Nelson, 1993); otros enfatizan en la importancia de la proximidad territorial para los sistemas nacionales y locales de innovación (Becattini, 1989; Garofoli, 1995; Storper, 1997); otros emplean la noción de innovative millieu (medio innovador) (Aydalot, 1986; Camagni, 1991; Maillat y Lecoq, 1992) en la investigación de las distintas dinámicas territoriales; y un creciente cuerpo de trabajos comienza a difundir la noción de learning region (región de aprendizaje) (Lundvall, 1995; Morgan, 1995, Maskell y Malmberg, 1995).

El concepto de sistemas y acuerdos productivos locales es principalmente un concepto heredado de Schumpeter (1934), Nelson y Winter (1982), y Freeman (1982), entre otros. Tal como menciona Amaral (2011, p. 3), esta perspectiva puede robustecerse con los conceptos del nuevo institucionalismo, en especial aquellas cuestiones relacionadas con las formas de coordinación y de gobernanza.

A pesar de los diferentes puntos de vista, Sousa (2004, p. 27) destaca que estos estudios comparten el reconocimiento de la importancia del contexto territorial tanto para los procesos de aprendizaje y creación de conocimiento como para el intercambio de conocimientos tácitos. Se establece una relación directa entre el éxito de determinados sistemas productivos y su capacidad para generar, incorporar y difundir innovaciones.

En Brasil, el énfasis en lo local llevó al desarrollo del concepto de Arranjos Produtivos Locais (Acuerdos Productivos Locales o APL) difundido por los investigadores asociados a La Red de Investigadores en Sistemas de Innovación y Producción Locales (RedeSIst) ${ }^{1}$.

Lastres (2007) distingue a los Sistemas Productivos e Innovativos Locales (SPIL) de los APL. Los SPIL se refieren a un conjunto de actores económicos, políticos y sociales que interactúan y se interrelacionan para la producción de determinados

RedeSIst: Rede de Pesquisa em Sistemas e Arrajos Productivos e Inovativos Locais.

Semestre Económico, volumen 16, №. 33, pp. 127-154 • ISSN 0120-6346, enero-junio de 2013, Medellín, Colombia 
bienes y servicios (industriales, agrícolas, culturales entre otros). En los SPIL se pueden observar lazos de cooperación e interacción entre los agentes que promueven la innovación. Por el contrario, en los APL no existen lazos significativos o cooperación entre los agentes, aunque prevalezca la concentración territorial de los mismos. Por lo tanto, los APL pueden ser sujetos de las políticas públicas para desarrollar tales lazos de cooperación entre los agentes.

El análisis de los sistemas de innovación, y su contrapartida local, los SPIL han conquistado de forma progresiva al ámbito académico en los últimos 15 años. Desde el ámbito de la política, su reconocimiento bajo la denominación de Acuerdos Productivos e Innovativos Locales (APIL), forma parte de un proceso que tiene su auge en Brasil, y cuya implementación en el resto del mundo es motivo de estudio. La producción e innovación se distinguen en el tiempo y en el espacio, como reflejo del carácter de asimilación y uso del conocimiento y las capacitaciones, lo que resulta en requerimientos específicos de políticas.

Según Alburquerque (2004, p. 2)

... la innovación tecnológica debe ser entendida en un sentido amplio, es decir, incluyendo los cambios e innovaciones sociales que acompañan y hacen viable el proceso de innovación. Ejemplos de innovaciones sociales son las nuevas alternativas y nuevos métodos de gestión de personal tales como la racionalización de las tareas laborales, las mejora en las condiciones de trabajo, el perfeccionamiento de los sistemas de motivación, la delegación de responsabilidades y competencias personales, entre otras.

La perspectiva territorial ha sido rescatada como forma de otorgar mayor efectividad a las políticas de producción e innovación, para situarlas en su locus real de implementación, lo que incrementa la posibilidad de generar sinergias y complementariedades. Cassiolato, Lastres y Stallivieri (2008, p. 30) argumentan que:

\footnotetext{
La articulación, atracción de actores y la integración de las acciones contribuyó a la ampliación del enfoque de APL más allá de las cuestiones de competitividad, innovación y sustentabilidad económica, tornando más evidentes los nexos de esos temas con cuestiones como: inclusión social, generación de empleo e ingreso, distribución de la tierra, desarrollo local, integración nacional y ocupación de fronteras.
}

Las políticas de promoción de SPIL brindan competitividad a las Pequeñas y Medianas Empresas (pymes), y por lo tanto son generadoras de empleo. De esta forma, según el MDICEB (2004, p. 8), se valoriza la cooperación, el aprendizaje colectivo, el conocimiento tácito y la innovación de las empresas e instituciones locales como cuestiones centrales para el aumento de la competitividad sustentable.

El objetivo de este trabajo es elaborar una revisión teórica del rol que cumplen las aglomeraciones productivas locales desde el punto de vista del desarrollo local, 
Acuerdos productivos desde la visión del desarrollo local: el rol de la innovación

con especial énfasis en su papel como promotoras de la innovación. Por tal motivo, la revisión teórica se focaliza en los SPIL estudiados por la RedeSist, en Brasil. Esta revisión contribuye a la discusión teórica y normativa para promover el desarrollo local en el resto de los países. La comprensión de los SPIL, a diferencia de los conceptos anteriores de cluster o distrito industrial, permite identificar los mecanismos que viabilizan o restringen la introducción de innovaciones en el territorio local y formular acciones de política.

El trabajo se organiza de la siguiente manera. En una primera sección, se ofrece un marco teórico que incluye en primer lugar, una breve revisión sobre la literatura de aglomeraciones productivas, que abarca desde cluster hasta los APIL. Luego, se analiza el concepto de desarrollo local. En una segunda sección, se brindan antecedentes teóricos sobre la relación entre las aglomeraciones productivas y el desarrollo local. En tercer lugar, se presentan algunas consideraciones sobre los estudios empíricos existentes en la materia. En cuarto lugar, se plantean las políticas de innovación resultantes de la nueva concepción de acuerdos productivos. Por último, se plasman algunas consideraciones finales.

\section{MARCO TEÓRICO}

\subsection{Concepto de cluster, distritos industriales y acuerdos productivos locales}

La literatura internacional enfatiza en forma creciente sobre la importancia espacial de las empresas para su competitividad. En este contexto espacial, una concentración sectorial o geográfica de empresas o de otros agentes económicos se conoce como cluster, aglomerado, acuerdo, red, polo productivo o distrito industrial.

Los clusters (Porter, 1990) son también conocidos como distritos industriales, (Becattini, 1989). La aglomeración es, en mayor parte, sectorial. El término se refiere a conglomeraciones de pymes localizadas en las proximidades de las grandes empresas. En este contexto, las pymes son beneficiadas por factores presentes en la economía local (infraestructura, mano de obra especializada, presencia de recursos naturales locales, etc.). Asimismo, las empresas se benefician de su proximidad geográfica, así como de su grado de interacción, junto con instituciones de la misma cadena productiva. Aunque existen diferentes tipos de redes, el concepto de cluster parece dominar entre las categorías relacionadas con el desarrollo local y regional.

La United Nations Industrial Development Organization (Unido) ofrece un programa de desarrollo de Clusters y Redes, que distingue entre ambos conceptos. En el ámbito del programa, los clusters son definidos como concentraciones sectoriales y geográficas de empresas que producen y venden una gama de productos relacionados o complementarios y, en consecuencia, enfrentan desafíos y oportunidades comunes. Por 
otro lado, Unido (2001, p. 9) define las redes como grupos de empresas que cooperan en un proyecto conjunto complementándose mutuamente y especializándose de manera de superar los problemas comunes, alcanzar la eficiencia colectiva y penetrar en mercados que serían inaccesibles a cada empresa de forma individual. Los clusters pueden ser considerados una etapa para el desarrollo de los acuerdos productivos.

La presencia de una red no implica necesariamente la proximidad geográfica de las empresas, ya que la cooperación y el aprendizaje colectivo pueden existir aun entre empresas que no están en la misma localidad. Sin embargo, se observa que la proximidad geográfica facilita la formación de redes sociales confiables, a través de las cuales fluye la información.

De acuerdo con Visser (2004, p. 12), la calidad de la gobernanza² difiere entre clusters en función de 4 variables: confianza, empresas líderes, intermediarios de conocimiento, y soluciones a los problemas de acción colectivos. Las empresas líderes tienen la habilidad y el incentivo de invertir en fuentes colectivas de competitividad. Luego, realizan inversiones con efectos externos positivos para el resto de las empresas del cluster. Pueden impulsar el progreso y la innovación en algunas redes de negocios específicas, explorar nuevos mercados y facilitar la internacionalización.

La especificidad territorial en el marco de la economía global implica ir más allá de la hipótesis de los distritos industriales; implica un nuevo concepto como el de sistema institucional territorial (Boscherini y Poma, 2000). El interés se centra más en los elementos de la dinámica externa al sistema productivo local, considerado en su conjunto, que en los elementos de la dinámica interna. Tal como señalan Boscherini y Poma (2000, p. 470):

Comprender las dinámicas del sistema productivo local como totalidad que interactúa significa reconocer el rol más amplio y dinámico que desempeñan las llamadas "externalidades territoriales" que tienen que abandonar su rol de "apoyo". El cambio territorial, por lo tanto, consiste en que los agentes territoriales dejan de ser "externalidades" para convertirse en "agentes" directos de la producción.

La versión brasilera de distritos y clusters se conoce como APL o Acuerdos Productivos Locales. Estos acuerdos productivos son aglomeraciones de pymes existentes en una localidad determinada. Son sistemas de producción que se encuentran enraizados en lo local, gracias a las ventajas competitivas que la localización proporciona. Las ventajas competitivas de localización surgen de la cooperación y de la posibilidad de perfeccionamiento del conocimiento. De esta manera, las pymes establecidas en estos territorios se vuelven más capacitadas y competitivas.

\footnotetext{
Según el Programa de Desarrollo de las Naciones Unidas (PNUD, 1998, p. 17), governance (gobernanza) es el ejercicio de la autoridad económica, política y administrativa para administrar los problemas de la sociedad. Desde el punto de vista corporativo, es el modo en que los directivos dirigen una empresa, y las reglas y costumbres que se aplican en esa dirección.
} 
Acuerdos productivos desde la visión del desarrollo local: el rol de la innovación

RedeSist, en Brasil, ha desarrollado junto con el concepto de APL el de SPIL para estudiar la relación entre grupos de empresas así como su vinculación con otros agentes (económicos, políticos y sociales) dentro de un territorio determinado. De esta manera, Cassiolato y Lastres (2003, p. 5) mencionan una propuesta de entendimiento de los sistemas productivos locales en los países en desarrollo, que se focaliza en un conjunto específico de actividades económicas que posibilite el análisis de interacciones, en particular aquellas que llevan a la introducción de nuevos productos y procesos.

Según el Servicio Brasilero de Apoyo a las Micro y Pequeñas Empresas (SEBRAE) son un fenómeno vinculado a las economías de aglomeración asociadas a la proximidad física de las empresas, en un mismo territorio, vinculadas entre sí por un flujo de bienes y servicios, que presentan especialización productiva y mantienen vínculos de articulación, interacción, cooperación y aprendizaje. Para su identificación son necesarios cuatro elementos: capital social³ , organización productiva, articulación político-institucional y articulación comercial.

Si bien existe una fuerte interacción entre los diferentes conceptos, estos no son necesariamente secuenciales. Los SPIL son un tipo más amplio de acuerdos productivos que los APL, en los cuales la interdependencia, articulación y vínculos consistentes resultan en interacción, cooperación y aprendizaje con potencial de generar el incremento de la innovación endógena, de la competitividad y del desarrollo local.

Lemos (2003) apunta las diferencias conceptuales entre APL y SPIL. Los APL pueden ser definidos como aglomeraciones de agentes económicos, políticos y sociales con foco en un conjunto específico de actividades económicas, que presentan vínculos e interdependencias. Por su parte, los SPIL son aquellos APL cuya interdependencia, articulación y vínculos consistentes resultan en interacción, cooperación y aprendizaje, lo que posibilita las innovaciones de productos, procesos y organizacionales, y, por lo tanto, la competitividad empresarial y capacitación social.

Según la visión de la RedeSist, y de acuerdo con las características del sistema productivo de Brasil, siempre que haya producción de cualquier bien o servicio habrá un acuerdo en torno a la misma, que involucra actividades y actores relacionados con la adquisición de materias primas, máquinas y demás insumos. Por otra parte, estos sistemas pueden variar desde los más rudimentarios hasta los más complejos y dinámicos.

3 Existen muchas definiciones del concepto. El capital social alude a las instituciones, relaciones y normas que conforman la calidad y cantidad de las interacciones sociales de una sociedad (Bourdieu, 1985; Coleman, 1988; Narayan y Pritchett, 1997; Putnam (1995). La eficacia y la eficiencia del capital social en los procesos de desarrollo están en relación directa con la consolidación de redes sociales confiables que le brinden al sujeto la posibilidad de desenvolverse plenamente.

Semestre Económico, volumen 16, №. 33, pp. 127-154 • ISSN 0120-6346, enero-junio de 2013, Medellín, Colombia 
El concepto de APL evoluciona desde la simple indicación de concentración industrial geográfica de pequeños y medios productores, para abarcar otras dimensiones tales como la territorialidad y especialización definidas en términos de cultura local, existencia de cooperación entre pymes y organización institucional, formas de gobernanza, aprendizaje colectivo, potencial para promover innovaciones y presencia de proveedores locales.

En el interior de los APL los procesos de aprendizaje implican la concreción de un conjunto de informaciones y conocimientos que son compartidos entre sus componentes, lo cual requiere, según argumentan Cassiolato, Brito y Vargas (2005), el montaje de códigos de lenguaje y canales de comunicación con el objetivo de visibilizar esta transferencia de manera eficaz.

De acuerdo con Moré, Lima y Nascimento de Almeida (2010, p. 193), el concepto de SPIL propuesto por la RedeSist se sustenta en una visión neo-schumpeteriana sobre innovación y cambios tecnológicos, que destaca los siguientes factores: a) la innovación y el conocimiento como elementos clave de la dinámica y del crecimiento de países, regiones, sectores, instituciones y organizaciones; b) la innovación como proceso de aprendizaje que depende de interacciones y sujeta al entorno ambiental; c) las diferencias en la capacidad de aprendizaje de los agentes; d) la existencia de especificidades en cada país, región, sector, organización; e) el rol del conocimiento tácito para el éxito innovativo y, al mismo tiempo, la dificultad de transferencia de esos activos.

El concepto de la RedeSist representa una unidad de análisis complementaria, y de ninguna manera sustitutiva de las demás. Suzigan y otros (2003, p. 12), al analizar los aspectos de especialización y concentración de empresas, proponen una tipología en el tratamiento de los diversos niveles de consolidación de los APL los cuales se presentan en el cuadro 1.

Cuadro 1: Tipología de SPIL de acuerdo a su importancia para una región

\begin{tabular}{|l|l|l|l|}
\hline \multicolumn{2}{|c|}{} & \multicolumn{2}{|l|}{ Importancia sectorial } \\
\cline { 3 - 4 } \multicolumn{2}{|l}{} & Reducida & Elevada \\
\hline \multirow{2}{*}{$\begin{array}{l}\text { Importancia } \\
\text { Local }\end{array}$} & Elevada & Vector de desarrollo local & $\begin{array}{l}\text { Núcleo de desarrollo sectorial } \\
\text { regional }\end{array}$ \\
\cline { 2 - 4 } & Reducida & Embrión de acuerdo productivo & Vectores avanzados \\
\hline
\end{tabular}

Fuente: Suzigan y otros (2003, p. 12).

Los APL que son importantes para la región, pero que no contribuyen de manera decisiva para el sector, son llamados vectores de desarrollo local. Aquellos acuerdos 
Acuerdos productivos desde la visión del desarrollo local: el rol de la innovación

que se destacan tanto por la elevada importancia local como sectorial, se convierten en núcleos de desarrollo sectorial regional. Cuando los APL poseen poca relevancia para el sector, y al mismo tiempo conviven con otras actividades económicas en la región, y se presentan con baja importancia local, son considerados embriones de acuerdo productivo. Existen, de otra forma, aquellos acuerdos que poseen elevada relevancia para el sector en relación con su participación en la producción o en el empleo, si bien insertos en un tejido económico mayor y más diversificado, con poco valor para el desarrollo local, y son denominados vectores avanzados.

\subsection{Desarrollo local: concepto y estrategias}

La teoría de desarrollo local consiste en un modelo de desarrollo que no se basa simplemente en mensurar o medir las variables económicas, como tasas de interés, salarios, inflación y sus posibles impactos regionales. Con el objetivo de tratar las potencialidades de una región geográfica determinada, se deben tener en consideración, sobre todo, los recursos naturales existentes, la vocación y competencia de la mano de obra de la comunidad y factores socio-culturales, entre los cuales se destacan la cooperación entre empresas, tradiciones, costumbres, etc. Alburquerque (2004, prólogo) señala

Tal como define la OIT el Desarrollo Económico Local es "un proceso de desarrollo participativo que fomenta los acuerdos de colaboración entre los principales actores públicos y privados de un territorio, posibilitando el diseño y la puesta en práctica de una estrategia de desarrollo común a base de aprovechar los recursos y ventajas competitivas locales en el contexto global, con el objetivo final de crear empleo decente y estimular la actividad económica.

El reconocimiento de la importancia trascendental de las redes en la competitividad de los territorios es observada en las investigaciones socioeconómicas: la cooperación inter-firma permite la construcción de sistemas de negocios que buscan entornos competitivos y de innovación. Este proceso tiene lugar tras la adquisición de ventajas resultantes de ciertas externalidades territoriales derivadas de la aglomeración y, también, de la tradición de negocios territorial (Narváez, Fernández y Senior, 2008; Caravaca, González y Silva, 2003).

Tras una revisión del marco teórico basado en el desarrollo económico local, Vázquez (1998) se refiere a un desarrollo construido por la economía según la escuela de crecimiento endógeno (Lucas, 1988; Romer, 1990; Aghion y Howitt, 1992). Algunas de las conclusiones que esta perspectiva considera han sido incorporadas en teorías más recientes del desarrollo endógeno.

La perspectiva de crecimiento y la del desarrollo endógeno comparten la visión de que los sistemas productivos consisten de un grupo de factores materiales e 
inmateriales que permiten a las economías locales y regionales adoptar trayectorias diferentes hacia el crecimiento para el desarrollo y, por lo tanto, existe un espacio para la política regional e industrial.

Vazquez (1998) argumenta que la construcción de un sistema productivo local integrado por varias empresas (la mayoría pymes) especializadas en un único producto en un territorio promueve los intercambios en múltiples mercados. Según Narváez, Fernández y Senior (2008, p. 8), "esto provoca tanto la emergencia de economías de escala externas, pero internas a los sistemas productivos locales, como la reducción de los costos de transacción. Estas externalidades causan retornos crecientes, y en consecuencia, promueven el crecimiento económico territorial". Narváez, Fernández y Senior (2008, p. 83) argumentan que la corriente del desarrollo local asume la importancia de los sistemas productivos locales para los procesos de crecimiento y los cambios estructurales en los territorios. Albuquerque (1997) establece que el desarrollo local puede ser entendido como un proceso de transformación de la economía y de la sociedad local para sobrellevar obstáculos y desafíos, y mejora la calidad de vida de los habitantes por medio de agentes socioeconómicos locales (públicos y privados) cuya actividad busca un uso más eficiente y sustentable de los recursos. Albuquerque (2004) rescata la importancia de considerar el desarrollo como un proceso, en lugar de un resultado.

Con respecto al desarrollo local, Porter (1990) se cuestiona por qué hay concentraciones exitosas de actividades económicas en algunos distritos que son relativamente pequeños, y no en otros. El autor observa que las empresas no se crean de manera independiente, sino que su desarrollo está condicionado por el entorno nacional que apoya y promueve la competitividad. Si en el sistema social existe algún factor de aceleración, entonces hay que identificar, otros elementos clave para comprender los mecanismos que estimulan el desarrollo espontáneo y la competitividad a escala local.

Porter (1990) identifica la creación de un cluster o grupo de unidades de producción y proveedores alrededor de una industria o servicio particular como uno de los cuatro factores que propenden a la competitividad regional, lo que ha sido llamado el diamante de la ventaja competitiva en el desarrollo económico local. Basado en la consideración de estos factores, Ickis (1998, p. 3, Cap.13) ha identificado los obstáculos que pueden impedir el desarrollo económico local. Estos obstáculos podrían ser: a) una dependencia excesiva en los factores que generan las ventajas comparativas, b) la distancia de los consumidores (en el sentido espacial, pero también, y más importante, en términos de comunicación e información), c) la ignorancia de la posición relativa en términos del mercado y d) la integración hacia adelante inadecuada y falta de cooperación en la industria. 
Acuerdos productivos desde la visión del desarrollo local: el rol de la innovación

Al referirse al desarrollo local y territorial, Gallicchio (2004, p. 6) menciona que se debe establecer la importancia de cuatro dimensiones: a) Económica: relacionada con la creación, acumulación y distribución de la riqueza; b) Social y cultural: implica calidad de vida, equidad e integración social; c) Ambiental: se refiere a los recursos naturales y a la sustentabilidad de los modelos de mediano y largo plazo y d) Política: gobernanza territorial, proyecto colectivo independiente y sustentable.

El desarrollo local supone un proceso de consenso entre actores que interactúan en un determinado territorio para impulsar a través de su participación un proyecto común de desarrollo. Implica generación de crecimiento económico, equidad, cambio social y cultural, sustentabilidad ecológica, enfoque de género y equilibrio territorial, entre otros aspectos. Es de naturaleza multidimensional, ya que, como se mencionara, inciden los aspectos económicos y sociales, y las condiciones ambientales, políticas y culturales.

El desarrollo local es un proceso orientado por la acción de diferentes agentes, y pocas veces emerge de forma espontánea. Esto requiere de una forma compleja de gobernanza, que involucre actores clave preocupados por el desarrollo de la región y sectores productivos seleccionados. En este sentido, los proyectos de desarrollo local involucran un gran número de actores institucionales y empresas, los cuales, de forma frecuente, no poseen mecanismos de coordinación preexistentes, y están inevitablemente bloqueados por patrones de interacción históricos. Según Street y Cameron (2007), las instituciones gubernamentales pueden actuar como intermediarios para promover el desarrollo de la confianza entre las partes interesadas

Narváez, Fernández y Senior (2008, p. 74) concluyen que por medio de la construcción de confianza, la promoción de la cooperación interfirma y la cooperación entre organizaciones públicas, privadas y las organizaciones comunitarias, la promoción de la especialización y de los procesos de innovación a través del uso compartido del conocimiento y de las competencias, las empresas y el performance del territorio pueden ser mejorados.

Un factor importante para el fomento del desarrollo local es la capitalización de la economía. Esto se vincula a la idea de accesibilidad a los mercados financieros, o de accesibilidad de los inversores locales al ahorro. Al respecto, Madoery (2005, p. 14) considera que el financiamiento que disponen los gobiernos locales para apoyar procesos de desarrollo es un aspecto fundamental.

Existen evidencias de que la promoción de emprendedores es una característica elemental de las estrategias de desarrollo local. Para aprovechar las capacidades emprendedoras se deben generar capacidades ambientales favorables. Con el 
objetivo de endogeneizar una variable tradicionalmente exógena, los planes de desarrollo local constan de incubadoras de empresas o vivero de empresas, agentes de desarrollo local, etc.

Como mencionan Lopera y Posada (2009, p. 121), los actores gestionan el desarrollo en su localidad y, según sus capacidades, generan cambios que diferencian unos espacios sociales de otros, en relación con los recursos naturales y técnicos, con la dotación en infraestructura, con los procesos de participación y de autogestión

Asimismo, Pérez y Carrillo (2000, p. 83) destacan que el fomento del crecimiento de la población activa ha de ser incorporado como una de las estrategias de desarrollo local. Esto implica introducir nuevas relaciones laborales, nuevas tecnologías (TIC) que facilitan el trabajo a distancia. La nueva concepción medioambiental y la importancia del sector turismo surgen como oportunidad para la integración laboral y el crecimiento de la población activa. No sólo el crecimiento de la población activa sino también su formación o acumulación de capital humano es fundamental para el desarrollo local. Por otro parte, destacan el papel de la Administración Pública en el desarrollo local. Las administraciones de ámbito inferior al nacional pasan a ser actores fundamentales de la prestación de servicios públicos. La educación y la sanidad se descentralizan para evitar las desigualdades y para prestar unos niveles de servicio más acordes con las necesidades de los ciudadanos de cada territorio. Esta descentralización aumenta las posibilidades de desarrollo local. En los últimos anos, las diferentes instancias de gobierno han avanzado en la preocupación por la cuestión local. Sin embargo, las iniciativas existentes son aún aisladas y esporádicas.

\section{ANTECEDENTES SOBRE AGLOMERACIONES PRODUCTIVAS, INNOVACIÓN Y DESARROLLO TERRITORIAL}

Esta sección toma como referencia la tipología de análisis de las aglomeraciones productivas de Vasconcellos (2007). Según la autora, la literatura sobre crecimiento y desarrollo económico evolucionó de manera significativa, durante el siglo XX, a partir de las publicaciones de Isard (1956), Location and Space Economy de la vertiente neoclásica. Isard, interesado en los análisis urbanos y regionales, procura incorporar en su teoría general las demás disciplinas de las ciencias sociales, sobre todo, la contribución de la geografía económica de Von Thünen, Lösch y Weber, preocupados por la localización espacial.

Otros analistas reconocen las contribuciones de los clásicos para la teoría de crecimiento económico, e incorporaron nuevas concepciones en lo que se conoce como economía del desarrollo (Schumpeter, 1934 y Hirschman, 1958) con importantes contribuciones para el tema regional. Examinaron las dinámicas alternativas 
Acuerdos productivos desde la visión del desarrollo local: el rol de la innovación

del desarrollo como por ejemplo el desarrollo en contraposición al subdesarrollo (Rostow, 1960), pobreza y dualidad (Nurkse, 1952 y Lewis, 1955), la problemática centro-periferia (Richardson, 1986), las condiciones desiguales de los términos de intercambio en el comercio internacional, entre otras. Ligados a las cuestiones de desarrollo espacial, figuran Myrdal (1957) y Hirschman (1958), y diferentes pensadores de la Comisión Económica para América Latina y el Caribe (CEPAL). El Estado debe intervenir para contener las fuerzas del mercado que tenderían, de lo contrario, a acentuar las desigualdades regionales. Estas cuestiones fueron, de alguna manera, introducidas en las reflexiones sobre desarrollo como un proceso endógeno, reflexiones en las que se destaca Sachs (1974). Fischer (2002) examina las experiencias y proposiciones sobre el desarrollo local en Brasil.

Por otro lado, se observan las contribuciones de la teoría institucionalista con la corriente neoinstitucionalista, que reconoce el papel de las instituciones en los sistemas económicos, basada en los principios neoclásicos. Aquí se destaca la idea de gobernanza (mercado, jerarquía, híbridos) y el concepto de costos de transacción (Coase, 1937; Williamson 1975). Los costos de transacción pueden definirse como los necesarios para ordenar o crear y operar las instituciones, y garantizar la obediencia de las reglas.

El enfoque institucionalista considera la existencia de costes de transacción no solo en los intercambios que se producen en el mercado, sino también en los intercambios en el interior de las empresas y organizaciones. Aunque Williamson (1975) no tenía una preocupación particular sobre los temas regionales, la teoría de los costos de transacción fue incorporada en varios análisis sobre aglomeración productiva, en el contexto de la organización industrial. Por otra parte, la corriente nueva economía institucional evolucionista aporta ideas más orgánicas y evolucionistas, basadas en las nociones de Schumpeter (1934) (innovación y crecimiento económico), Simón (1955) (racionalidad limitada, comportamiento humano y organizacional) y Darwin (1859) (concepto de evolución, ambiente, selección). Consideran la presencia de un nuevo paradigma tecnológico, basado en las Nuevas Tecnologías de la Información y de la Comunicación (TIC). Entre sus exponentes se destacan Lundvall (1995), Nelson y Winter (1982). Además de sus aplicaciones en estudios intra e interfirmas, la perspectiva evolucionista, como es el caso de la escuela californiana, ha sido utilizada como referencia en importante estudios sobre desarrollo local.

En la literatura clásica sobre desarrollo uno de los conceptos fundamentales de región es derivado de las contribuciones de Perroux (1967) sobre las tres categorías de espacio económico y región económica. Ellas son: a) espacio económico como un agregado homogéneo constituido por un conjunto de elementos con caracte- 
rísticas semejantes, b) espacio polarizado, heterogéneo, en que las diversas partes son complementarias y mantienen sistemas de cambio entre sí y sobre todo con el polo dominante, c) espacio definido por un plan o programa de acción, cuando sus partes dependen de un centro de decisión o de un programa.

Por otro lado, se destaca el aporte de Marshall (1975), entre otros, sobre la noción de distrito industrial. La idea de que se gana en la formación de aglomeraciones sectoriales en determinado espacio geográfico fue introducida en la economía industrial por Alfred Marshall (1890) en su obra Principios de economía. Las localidades fueron denominadas industria localizada o distritos industriales.

Según Marshall (1975) las ventajas económicas (externalidades positivas) que pueden ser obtenidas por las empresas que pertenecen a una localidad donde predomina un sector específico surgen tras el fácil acceso a trabajadores calificados (por la concentración local de mano de obra especializada), los proveedores de materias primas y servicios relacionados con la actividad principal, y que contribuye para crear un ambiente propicio a las innovaciones.

Las nuevas ideas sobre la existencia de sistemas de pymes interrelacionadas a través de redes se fundamentaron originariamente en la obra de Piore y Sabel (1984), La segunda ruptura industrial. El artículo de Becattini (1989) brinda rigurosidad científica al estudio de los distritos industriales.

A diferencia del distrito industrial y de su posterior generalización en la noción de sistema productivo local, el medio innovador no privilegia la visión social, y sí la tecnología, considerándola especial. Sin embargo, el principal foco de la mayor parte de las contribuciones que integran la perspectiva sobre medio innovador recae en la naturaleza de los procesos de aprendizaje interactivo que dan origen a las innovaciones en el ámbito de las aglomeraciones productivas. La empresa no es considerada un agente aislado en el proceso de innovación, sino parte de un ambiente innovativo.

Según Dias da Rocha (2005, p. 46), los procesos de innovación involucran de forma frecuente la participación y consecuente coordinación de varias organizaciones, que están sujetas a path dependency5 de determinado tipo, en un momento determinado. El Centre for Research on Innovation and Competition (CRIC) ) enfatiza las interacciones e interdependencias entre las organizaciones, basadas en relaciones

4 El Millieu innovativo es descripto como un conjunto de elementos materiales (empresas, infraestructura), inmateriales (conocimiento) e institucionales (reglas y normas legales) que componen una compleja red de relaciones orientadas a la innovación.

5 Desarrollo pasados que influyen y limitan los nuevos avances. 
Acuerdos productivos desde la visión del desarrollo local: el rol de la innovación

socialmente enraizadas que tienen un papel importante en el diseño de los procesos de producción e innovación. Tales relaciones son estructuradas en torno a tres espacios funcionales distintos que incluyen: el espacio de producción, el espacio de mercado, y el espacio de apoyo (instituciones). Es el espacio de apoyo el que permite a las empresas enfrentar las incertidumbres inherentes a los ambientes de competencia en la medida que determina las relaciones entre la capacidad de innovación de las empresas y el desarrollo territorial. Como expone Granovetter (1985) las relaciones entre los actores están embebidas en relaciones sociales.

Asimismo, los trabajos de Porter (1990) y Storper (1997), alineados con la corriente de organización industrial, repercutirán de manera positiva en el ámbito de los estudios organizacionales, y se desplaza el foco de interés de la empresa hacia ciertos territorios caracterizados como clusters. Para Storper (1997, p. 3), por ejemplo, una región debería vislumbrarse no necesariamente como el resultado de un intenso proceso económico y político, sino más bien como una unidad fundamental de la vida social equivalente, por ejemplo, al mercado, al Estado o la familia, como un motor generador del proceso de vida social. Algunas dimensiones del concepto de Storper guardan sintonía con el concepto de territorio. Según Storper (1997, p.286) los sistemas productivos más dinámicos caracterizados por las innovaciones emergen de racionalidades endógenas y diferenciadas de actores colectivos ligados entre sí por sus propias convenciones, frecuentemente de base local.

Antes de que los APL se conviertan en un área de conocimiento tan importante en economía industrial, los estudios sobre la competitividad de las empresas estaban focalizados en su mayoría en cuestiones tecnológicas, de demanda, escala, financiera, así como también en políticas sectoriales. La política industrial seguía los mismos principios.

En la economía industrial, la cuestión de la localización era vista principalmente por sus aspectos de costos de transporte y de acceso a los insumos y servicios. De la misma manera, la economía regional estaba focalizada en cuestiones de infraestructura, urbanización, costos de transporte, entre otros, lo que lo convertía en un enfoque de base regional y urbana. Las cuestiones locales concernientes con las relaciones de las pymes, el espacio y el conocimiento no eran tratadas. La política de desarrollo regional seguía estos principios. La creciente importancia dada a la cuestión local surge tras la experiencia de desarrollo económico ocurrida en la Tercera Italia y en el Valle de Silicio, en Estado Unidos.

Por otro lado, las corrientes asociadas a la temática de Sistema Nacional de Innovación (Freeman, 1987; Lundvall, 1995; Nelson, 1993) dieron origen al concepto de SPIL. La teoría de los Sistemas Nacionales de Innovación (Lundvall, 1995) se 
focaliza en los actores, las redes, y las interacciones, donde la innovación se constituye como un indicador de la calidad de esas interacciones. Más recientemente, esta teoría ha adoptado una perspectiva más flexible respecto a los niveles en los cuales operan los sistemas de innovación, lo que da origen a los sistemas regionales o locales de innovación.

Gráfico 1. Innovación y desarrollo territorial: principales teorías interpretativas

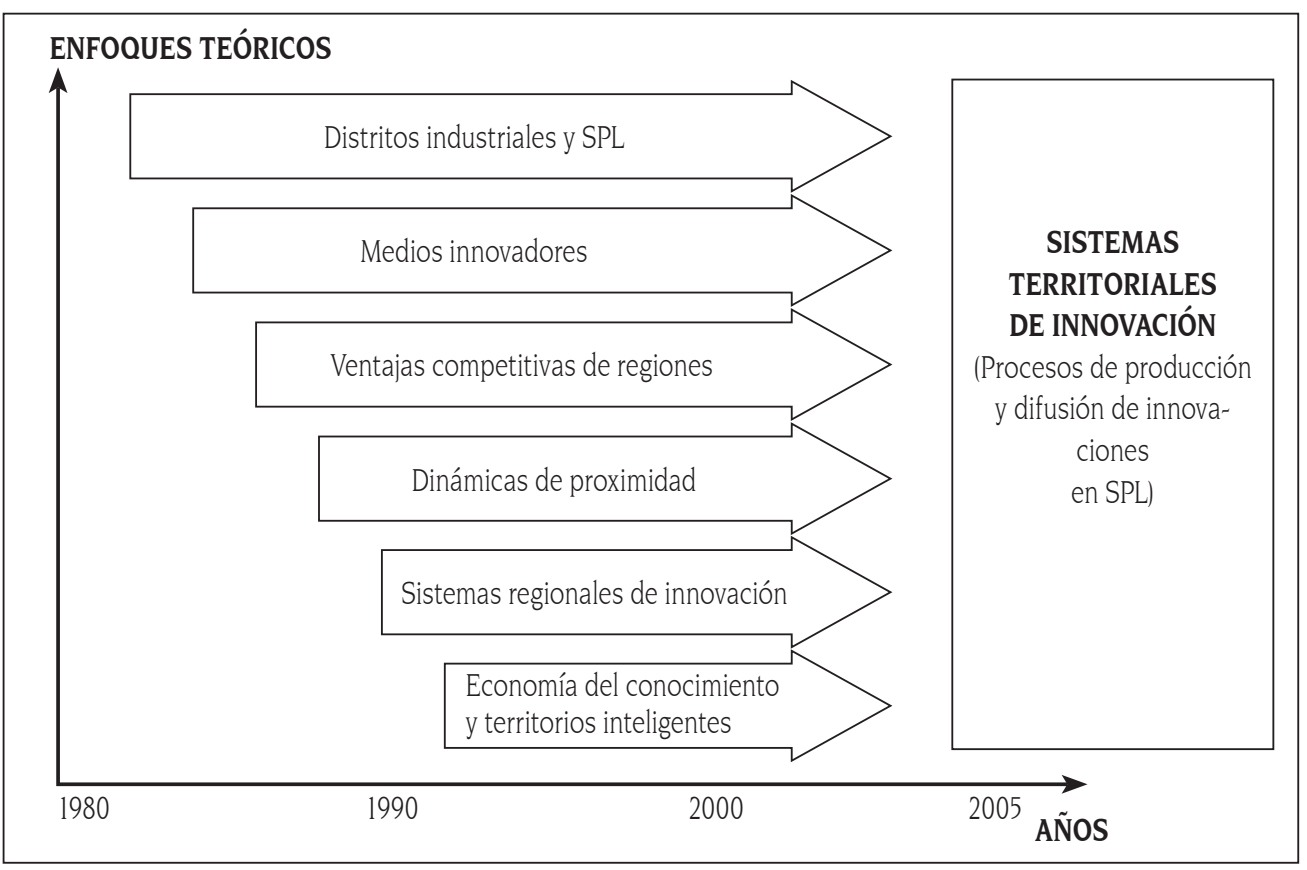

Fuente: Méndez, (2006, p. 7)

Lundvall (1995) reconoce la innovación como un proceso social interactivo que ocurre en una dinámica de acción colectiva, fuertemente enraizada en las estructuras económicas y sociales. Se introduce la idea del aprendizaje por interacción, donde las empresas actúan en un ambiente complejo y de incertidumbre. Desde la perspectiva del aprendizaje (Lundvall, 1995) cuando dos empresas cooperan, intercambian información, logran conocerse mejor y así construyen la confianza. Este es el fundamento clave para fomentar diversas modalidades de aprendizaje, con lo cual se apuesta a que el aprendizaje no es solo un proceso técnico sino social. Sin embargo, algunos autores como Núñez, Montalvo y Pérez (2006, p. 177) consideran que esta teoría tiene como característica el énfasis en las empresas como agentes de innovación y en la dimensión económica de la innovación. Por lo tanto, se debe abordar la innovación desde una manera más integral que abarque 
Acuerdos productivos desde la visión del desarrollo local: el rol de la innovación

consideraciones sociales, políticas y ambientales donde participen los distintos actores del desarrollo local.

Méndez (2006) realiza un esquema sobre la evolución teórica de la relación entre territorio e innovación, desde el concepto de distrito industrial hacia la noción de territorios inteligentes.

\section{ESTUDIOS EMPÍRICOS SOBRE ACUERDOS PRODUCTIVOS Y DE INNOVACIÓN LOCALES}

Mediante una revisión bibliográfica respecto al objeto de estudio, se observa un predominio de trabajos que brindan contribuciones teóricas comparadas a la cantidad de investigaciones empíricas. Tal como expresan Arocena y Sutz (2006, p. 257):

La conceptualización de los procesos de innovación ha tenido lugar a partir de estudios empíricos que, entre otros aspectos, pusieron de manifiesto el carácter "sistémico" de la innovación en los países desarrollados. En ciertos casos, ese carácter llega a expresarse en un conjunto relativamente estable e "institucionalizado" de relaciones entre diversos organismos -empresas, agencias gubernamentales, centros de investigación, bancos, entidades gremiales, etcétera- que constituyen así un "sistema", como el que Freeman (1987) analizó en un estudio sobre Japón que constituye uno de los cimientos de la teoría de los sistemas innovativos.

Arocena y Sutz (2006, p. 257) señalan que cuando se estudia la innovación "realmente existente" en los países subdesarrollados es probable que lo primero que impacte sea su carácter informal. En los países menos desarrollados, no se puede dar por supuesto que la innovación tenga carácter sistémico. Esta se realiza a través de vínculos e interacciones entre actores diversos, pero unos y otros suelen ser frágiles, episódicos y escasos. Sin embargo, Arocena y Sutz (2006, p. 258) argumentan que "pese a ello, un grado sustancial de innovación ha existido y existe en América Latina. Los trabajos de RedeSist lo han puesto claramente de manifiesto en el caso de Brasil".

Si bien los estudios empíricos referidos a la innovación estuvieron centrados en los países desarrollados, es posible hallar un grado importante de innovación en América Latina. En el caso de Brasil, se ha puesto de manifiesto a partir de los SPIL.

Cassiolato, Lastres y Martins (2000) realizan un estudio empírico sobre sistemas de innovación en quince casos de estudio, referidos a APL en Brasil, Uruguay y Argentina. En Brasil se analizaron cuatro acuerdos agroindustriales: tabaco y elaboración de vino en Río Grande Do Sul; chocolate y cacao en Bahía; frutas tropicales en el nordeste de Brasil, así como cinco casos de cluster tecnológicos como biotecnología en Minas Gerais; software, en Río de Janeiro, telecomunicaciones, en Campinas y Paraná, entre otros. Por su parte, se incluyeron el cluster de producción de vino en 
Uruguay y tres casos de Argentina de las localidades de Tres de Febrero en el Gran Buenos Aires, Rafaela y Mar del Plata.

Cassiolato y Szapiro (2003) ofrecen una tipología de aglomerados económicos, a partir del análisis empírico de 26 APL en Brasil a partir del cual se muestra la relevancia de los elementos de gobernanza y la presencia de redes territoriales en la creación de condiciones de enraizamiento para la innovación local. Texeira y Ferraro (2009, p. 22) mencionan que "sin embargo, no todas las aglomeraciones productivas poseen estos tipos de ventajas locativas. En efecto, los procesos históricos de formación de algunas aglomeraciones no alcanzaron un grado de desarrollo que incluya el aprendizaje activo y la innovación entre sus actividades." Pese a poder encontrar ciertas generalidades, el tipo de innovación y su evolución dependen del sector. Por ejemplo, los cluster de frutas y de producción de vinos se basan en pymes domésticas, donde el mercado local es importante, pese al incremento del mercado externo, y donde los centros de investigación juegan un rol en el desarrollo de innovaciones, e importa la innovación en la experimentación y poco, en la formalización de actividades como IED. Por el contrario, en el cluster tecnológico, la competencia se basa en la capacidad de diseño, el desarrollo de nuevos productos, etc., y una fuerte interacción con laboratorios de IED. La evolución del aprendizaje y de las capacidades de innovación de las pymes analizadas depende en gran medida del tipo de relaciones que establecían con otras empresas a lo largo de la cadena productiva y de cómo podían reaccionar a los cambios en la política.

Suzigan y otros (2003) avanzan en la elaboración de una propuesta que permite evaluar el grado de importancia que un aglomerado posee para el crecimiento económico de la localidad de pertenencia, tanto en términos de generación de empleo como de ingreso. De esta manera, los autores identifican las aglomeraciones de empresas que potencialmente pueden contribuir al desarrollo local.

Por otro lado, Moré, Lima y Nascimento de Almeida (2010) analizan el plan de desarrollo del APL de Moda íntima Friburgo, Brasil, y enfatizan en los factores que influyen en el desarrollo del SPIL, las innovaciones y las nuevas tecnologías de forma principal.

A diferencia del caso de los países desarrollados, en los países en desarrollo la idea de los sistemas de innovación está caracterizada por altos niveles de diversidad e inestabilidad institucional.

\section{NUEVAS POLÍTICAS DE INNOVACIÓN EN ACUERDOS DE PRODUCCIÓN}

Los argumentos teóricos plasmados a favor de la constitución de sistemas de producción e innovación locales enfatizan que la política para su conformación puede 
Acuerdos productivos desde la visión del desarrollo local: el rol de la innovación

ser tan eficiente como la política industrial y, por lo tanto, generadora de empleo, así como también puede contribuir al desarrollo local al considerarse como políticas regionales y sociales.

Tal como expresan Lastres y Cassiolato (2003, p. 7), las nuevas políticas responden a un nuevo concepto de innovación y de relación entre las empresas y demás actores locales y regionales. Estas difieren radicalmente de las políticas anteriores basadas en la visión dicotómica y lineal de la innovación. En sus principios el concepto de innovación se basaba en una visión funcional y jerarquizada del conocimiento (ciencia, tecnología, innovación) y su difusión a través de las instituciones de Ciencia y Tecnología. Como afirman Lastres y Cassiolato (2003, p. 8), tales políticas se concentraban en apoyar el lado de la oferta o de la demanda de las tecnologías, como si las mismas fuesen alternativas excluyentes.

Por su parte, las nuevas políticas de innovación buscan:

- Estimular las múltiples fuentes de conocimiento, así como las interacciones entre los diferentes agentes.

- Fomentar la difusión del conocimiento codificado y tácito para toda la red de agentes locales.

- Formar el recurso humano en función de las necesidades de innovación de los diferentes sistemas de producción locales.

- Fomentar la interacción creativa entre los actores públicos y privados a través de redes, con el objetivo de crear el marco institucional adecuado y un entorno territorial innovador (Albuquerque, 2004) que facilite el acceso a los servicios de desarrollo empresarial para las pymes locales.

- Otorgar importancia a la dimensión territorial de la política tecnológica para facilitar el fomento de las potencialidades endógenas de cada zona.

Por otro lado, Lastres y Cassiolato $(2003$, p. 8) afirman que las políticas dirigidas a la promoción de la interacción entre actores de ninguna manera sustituyen las acciones de apoyo público a la infraestructura científica y tecnológica.

El primer desafío desde el punto de vista de la política consiste en cambiar la lógica de actuación individual de varios organismos gubernamentales vinculados a los temas de desarrollo local y regional. Resulta evidente que ningún órgano o institución, de forma individual, posee la capacidad de enfrentar el desafío del desarrollo territorial de una manera integral. 
Al mismo tiempo, se busca complementar con el abordaje de los acuerdos de producción locales los esfuerzos de aumento de competitividad de las diversas cadenas productivas de varios órganos de gobierno e instituciones de apoyo.

En América Latina, los organismos internacionales como el Banco Interamericano de Desarrollo (BID) y el Banco Mundial (BM) incentivan la consolidación de clusters a través de la promoción de programas de competitividad regional. Además, los pro yectos de UNIDO han estimulado innovaciones de productos, procesos y funciones productivas, así como han facilitado el acceso a nuevos mercados y contribuido a la creación y fortalecimiento de las instituciones (a través de la construcción de normas colectivas y organizaciones).

En Brasil, las políticas de apoyo a los APL consisten en:

- Identificar los APL existentes en el país, inclusive aquellos territorios que presentan potencialidad para constituirse como futuros APL, de acuerdo con su importancia en el respectivo territorio.

- Definir criterios de acción conjunta gubernamental para el apoyo y fortalecimiento de los APL, con respeto hacia las especificidades de actuación de cada institución y que estimulen el asociativismo, la sinergia y la complementariedad de las acciones.

Según el MDICEB (2004), las políticas de fomento de las pymes son más efectivas cuando están dirigidas a grupos de empresas y no a empresas individuales. Surge una nueva percepción que se refleja a través de las políticas públicas de desarrollo, en que la dimensión local pasa a ser tratada como un eje orientador de promoción económica y social. Para los casos en que no haya suficientes condiciones para la elaboración del plan de desarrollo, la política pública deberá actuar en el estímulo de las condiciones mínimas que permitan su elaboración, con apoyo a la construcción de canales de interacción de los agentes involucrados y al surgimiento de líderes locales que puedan coordinar los diversos intereses en torno de una propuesta común.

Por otro lado, de acuerdo con la definición de sistema productivo local adoptada por la RedeSist el número de sistemas de producción locales existentes, por ejemplo, en Brasil es tan grande como la capacidad productiva brasileña lo permita. Este entendimiento hace más difícil la estructuración e implementación de políticas, en la medida que presupone un conocimiento profundo de cada caso. Desde el punto de vista normativo, no basta con desarrollar indicadores y mapas que busquen identificar la cantidad de sistemas existentes y sus diferentes configuraciones y grados de desarrollo. Según señalan Lastres y Cassiolato (2006, p. 120) por estar basadas en el reconocimiento de las especificidades de los diferentes sistemas, las 
Acuerdos productivos desde la visión del desarrollo local: el rol de la innovación

políticas para su promoción son incompatibles con modelos genéricos que utilizan ideas de benchmark y best practice. Tal como expresa el BNDES (2004), en las ciudades donde los APL son parte importante de la economía, la política parece ser realizada de manera mucho más consensual. Es una política donde existen, como referencias constantes, algunos principios e intereses comunes.

Si bien el objetivo de este trabajo no consiste en realizar un análisis comparativo, en Argentina así como en otros países, a diferencia de Brasil, los actores institucionales no están presentes siempre que exista producción de un bien o servicio, con lo cual no existe multiplicidad de casos de acuerdos productivos locales como en Brasil. Los acuerdos existentes reconocidos son pocos. La especificidad de los mismos viene vinculada a su individualidad y escasez. En Brasil, si se observan los mapeamientos realizados, en todos los territorios es posible encontrar un ejemplo de APL. En Argentina este no es el caso. Por lo tanto, cobra mayor sentido promover políticas para determinar su grado de desarrollo y el papel que juegan en ellos los actores institucionales, así como establecer el vínculo entre los clúster de producción y su contribución al desarrollo local.

De acuerdo con Reinert y Reinert (2003), a pesar de las ventajas ofrecidas por el análisis de los SPIL, han sido realizadas algunas críticas a las nuevas políticas formuladas en principio para promover tales sistemas al caracterizarlas como "a thin icing on a solid neo-classical cake" (un fino glaseado sobre una sólida torta neoclásica).

Es posible indicar algunos caminos para que las políticas y acciones estén dirigidas a la promoción de la innovación regional, sin olvidar que el concepto de innovación aplicado desde la perspectiva de los acuerdos de producción e innovación locales reconoce el carácter sistémico y complejo del proceso innovacción.

De acuerdo con Lastres y Cassiolato (2006, p. 120) detrás de cualquiera de los modismos actuales que enfatizaron desde la creación de polos y parques, incubadoras, distritos industriales, APL o sistemas de innovación, hay concepciones bastante interesantes, que muchas veces fueron desconsideradas en la ansiedad de implementar acciones rápidas. Es necesario recordar también que la incorporación de nuevos conceptos, modelos e instrumentos está lejos de ser trivial y demanda cambios de cultura, así como un importante aprendizaje institucional, principalmente por parte de investigadores, planificadores y hacedores de política, o sea, de las agencias e individuos encargados de desarrollar, implementar y evaluar las políticas.

Es importante mencionar que, a pesar de que existen argumentos fuertes a favor de este tipo de intervención pública, no hay una receta eficaz para el diseño de los instrumentos de promoción de conglomerados, ni garantías con respecto a 
los resultados de su aplicación. En consecuencia, existen riesgos e incertidumbres ligados al éxito de este tipo de política industrial (Texeira y Ferraro, 2009).

Desde el punto de vista operativo, disponer de algunas herramientas estadísticas y conceptuales facilitadoras en el ámbito local es un desafío, si se tiene en cuenta la carencia de información estadística sustantiva sobre el alcance y contenido de los sistemas de producción territoriales o los mercados de trabajo locales. Como expresa Alburquerque (2004a, p. 2):

\begin{abstract}
Es muy importante esta atención hacia los datos del nivel local y sobre las redes o tramas productivas empresariales ya que la carencia de información con la que hay que operar en los territorios dificulta siempre los esfuerzos para la elaboración de estrategias de desarrollo económico local. En efecto, las estadísticas convencionales se centran fundamentalmente en resultados agregados de la actividad económica o bien en una información sectorial que impide una visión integrada de las tramas productivas reales en los territorios concretos donde ellas se encuentran.
\end{abstract}

\title{
5. CONSIDERACIONES FINALES
}

A través de una revisión teórica se abordan los fenómenos de aglomeraciones productivas, innovación y desarrollo local desde la perspectiva de los sistemas de producción e innovación locales. La comprensión de los mismos, a diferencia de los conceptos anteriores de cluster o distrito industrial, permite identificar los mecanismos que viabilizan o restringen la introducción de innovaciones en el territorio local y formular acciones de política.

La mayoría de los estudios sobre producción e innovación locales se han centra do en el análisis de los países desarrollados, como el caso de los distritos industriales italianos, y pocos se han centrado en el estudio de los países en desarrollo.

El concepto de innovación ha evolucionado en el tiempo desde una visión que centraba el análisis en la empresa innovadora hacia nuevos enfoques que rescatan el rol del entorno territorial para su desarrollo. En torno a estas visiones, también han evolucionado las políticas públicas de apoyo a la innovación y al desarrollo local. De cualquier manera, el fenómeno de la innovación no debe atribuirse únicamente al contexto, sino, más bien, es precisa una visión más amplia para su tratamiento, que aborde el análisis más allá de la empresa y del aglomerado para lograr una interpretación de las diferencias en el comportamiento innovador de los territorios.

Para un adecuado aprovechamiento de las innovaciones, es conveniente que los agentes que las introducen, desde las empresas hasta las instituciones y organismos gubernamentales, hayan previamente efectuado los cambios internos 
Acuerdos productivos desde la visión del desarrollo local: el rol de la innovación

y organizacionales necesarios. Como indican varios autores, es necesaria la presencia de una estructura de gobernanza que brinde cierta organización, ya que las innovaciones no tienen impacto por sí mismas, sino por el sistema en el que están insertas.

Por otro lado, con el transcurso del tiempo, la innovación acompaña el desarrollo cada vez más complejo de las interacciones entre las empresas y las instituciones, plasmado en la evolución de las aglomeraciones productivas. De esta manera, las aglomeraciones productivas se convierten en sistemas de innovación más complejos que los distritos o clusters, donde el territorio ofrece ciertos activos, tanto tangibles como intangibles necesarios para la innovación y que propenden al desarrollo local. Como expresa Méndez (2006, p.2) "también convergen en la creciente atención prestada al territorio, no como simple escenario inerte, sino como acumulación histórica de recursos, actores y relaciones socioeconómicas con características diversas, que condicionan de forma positiva 0 negativa los procesos de innovación y desarrollo".

Uno de los factores esenciales para el desarrollo de las aglomeraciones locales caracterizadas por la producción y la innovación es el apoyo de los gobiernos. Más aún teniendo en cuenta que las aglomeraciones productivas bien estructuradas como los APL poseen un gran impacto en el desarrollo económico y social de las regiones donde se instalan. Por este motivo, puede considerarse que la promoción de este tipo de configuraciones productivas puede resultar en un instrumento de política regional y social.

En los países en desarrollo, las políticas de innovación tendrían que contemplar que los sistemas de innovación no se desarrollan ni funcionan por sí mismos ni aisladamente. Sin embargo, se han mencionado en este artículo algunas experiencias dentro de América Latina, como es el caso de Brasil, donde las políticas de innovación apoyan la creación y en algunos casos la supervivencia de los aglomerados de producción e innovación.

En Argentina, donde la identificación y presencia de los acuerdos de producción e innovación no es ni tan evidente ni tan amplia como en el caso de Brasil, cobraría mayor sentido promover políticas para determinar su grado de desarrollo y el papel que juegan en ellos los actores institucionales, así como establecer el vínculo entre los acuerdo productivos y su contribución al desarrollo local.

La trayectoria de desarrollo de un aglomerado productivo es influenciada por las políticas públicas, por el marco institucional y por la infraestructura de apoyo en el ámbito nacional. Restaría a futuro poner atención en cómo el desarrollo es influenciado por los acuerdos productivos en ellos insertos. 


\section{BIBLIOGRAFÍA}

Aghion, P. y Howitt, P. (1992). A model of growth through creative destruction. En: Econometrica, vol. 60, N. $^{\circ} 2$, p. 323-351.

Albuquerque, F. (2004). Desarrollo económico local y descentralización en América Latina. En: Revista de la CEPAL, N. ${ }^{\circ}$ 82, p. 157-171.

Alburquerque, F. (1997). Desarrollo económico local y distribución del progreso técnico. Una respuesta a las exigencias del ajuste estructural. En: Cuadernos ILPES, Vol. 43, p. 1-118.

Amaral Filho, J. (2011). Sistemas e arranjos produtivos locais. En: Planejamento e políticas públicas, N. $\left.{ }^{\circ} 36\right)$, enero/junio, p. 172-212.

Arocena, R. y Sutz, J. (2006). El estudio de la innovación desde el Sur y las perspectivas de un nuevo desarrollo. En: Gallina, A.; Núñez Jover, J.; Capecchi, V. y Arriete, L. Innovaciones creativas y desarrollo humano. Proyecto ALFA Lentisco. Italia. Ediciones Trilce, p. 255-275.

Aydalot, P. (1986). Milieux Innovateurs en Europe. Paris, GREMI, 361 p.

Becattini, G. (1989). Modelli locali di sviluppo. Bolinia, II Mulino, 231 p.

BNDES (2004). Arranjos produtivos locais como instrumento de desenvolvimento. Publicación Banco Nacional Do Desenvolvimento, Brasil, 78p.

Bourdieu, P. (1985). The forms of capital. En: Richardson, J. G. Handbook of Theory and Research for the Sociology of Education. Greenwood Press, Nueva York, p. 241-258.

Boscherini, F. y Poma, L. (2000). Más allá de los distritos industriales, pp. 23-38. En: Poma, L. Boscherini, F. y Yoguel, G. (Eds) Territorio, conocimiento y competitividad de las empresas: el rol de las instituciones en el espacio global. Dávila editores, 440p.

Camagni, R. (1991). Local 'Milieu', Uncertainty and Innovation Networks: Towards a New Dynamic Theory of Economic Space, pp. 121 142. En R. Camagni (ed.), Innovation Networks: Spatial Perspectives, Belhaven Press, Londres, 224p.

Caravaca, I; González, G. y Silva, R. (2005). Innovación, redes, recursos patrimoniales y desarrollo territorial. En: Revista Eure, Vol. 31, N. 94 , p. 5-24.

Caravaca, I.; González, G. y Silva, R. (2003). Redes e innovación socio-institucional en sistemas productivos locales. En: Boletín de la Asociación de Geógrafos Españoles, N. 36, p. 103-115.

Cassiolato, J.E; Lastres, H.M. y Stallivieri, F. (2008). Arranjos Produtivos Locais: uma alternativa para o desenvolvimento. Volume 2: Experiencias de Políticas. E-papers Servicos Editoriais Ltda, Rio de Janeiro, Brasil, 373p.

Cassiolato, J.E.; Britto, J. y Vargas, M. (2005). Arranjos cooperativos e inovação na indústria brasileira. En: De Negri, A.; Salerno, S. (org.) Inovações, padrões tecnológicos e desempenho das firmas industriais brasileiras. IPEA, Brasília, p. 511-577,

Cassiolato, J. E. y Lastres, H. M. M. (2003). O foco em arranjos produtivos e inovativos locais de micro e pequenas empresas, pp. 21-34. En: Lastres, H. M. M.; Cassiolato, J. E.; Maciel, M. L. (Orgs.). Pequena empresa: cooperação e desenvolvimento local. Relume Dumará, São Paulo, 208p. 
Acuerdos productivos desde la visión del desarrollo local: el rol de la innovación

Cassiolato, J. E, y Szapiro, M. (2003). Uma caracterização de Arranjos Produtivos Locais de Micro e Pequenas Emrpesas, p. 35-50. En: Lastres, H. M. M.; Cassiolato, J. E.; Maciel, M. L. (Orgs.). Pequena empresa: cooperação e desenvolvimento local. Relume Dumará, São Paulo, 208p.

Cassiolato, J; Lastres, H. y Martins, H. (2000). Local systems of innovation in Mercosur countries. En: Industry and Innovation, Vol. 7, N. ${ }^{\circ}$, p. 33-55.

Cavestany, F. (2000). Desarrollo desde el territorio. A propósito del desarrollo local. Universidad Nacional de Quilmes, Biblioteca digital. [En línea] http://biblioteca.municipios.unq.edu.ar/ modules/mislibros/archivos/Barreiro.pdf. Consultado 08-10-2012.

Coase, R. (1937). The nature of the firm. En: Económica, Vol. 4, N. 16, p. 386 405.

Coleman, J. (1988). Social capital in the creation of human capital. En: American Journal of Sociology, N. ${ }^{\circ}$ 94, p. 95-121.

Darwin, C. (1859). The origin of species by means of natural selection. London: John Murray, 502p.

Dias, Da Rocha, J.F. (2005). Construção Institucional da Qualidade em Produtos Tradicionais. Rio de Janeiro. Tesis de doctorado. Universidade Federal Rural do Rio de Janeiro. Instituto de Ciências Humanas e Sociais. Orientador: Professor Doutor John Wilkinson, 158p.

Fischer, T. (2002). Poderes locais, Desenvolvimento e Gestão: introdução a uma agenda, p. 12-32. En: Gestão do desenvolvimento e poderes locais: marcos teóricos e avaliação. Salvador: Casa da Qualidade, 341p.

Freeman, C. (1982). The economics of industrial innovation. Pinter, Londres, 470 pp.

Freeman, C. (1987). Technology policy and economic performance: lessons from Japan. London: Pinter, $155 \mathrm{pp}$.

Freeman, C. (1988). The factory of the future, the productivity paradox, japanese just-in-time and information technology. Economic and Social Research Council, PICT Policy Research Papers, N. 3 , Londres, 26 pp.

Gallicchio, E. (2004). El desarrollo local en América Latina. Estrategia política basada en la construcción de capital social. Seminario Desarrollo con inclusión y equidad: sus implicancias desde lo Local, SEHAS, Córdoba (Argentina), 24 pp.

Garofoli, G. (1995). Desarrollo económico, organización de la producción y territorio, pp. 53-71. En: Vázquez Barquero, A. y Garofoli, G. (editores). Desarrollo Económico Local en Europa, Colegio de Economistas de Madrid, Madrid, 281 pp.

Granovetter, M. (1985). Economic action and social structure: The problem of embeddedness. En: The American Journal of Sociology, Vol. 91, N. ${ }^{\circ}$ 3, pp. 481-510.

Hirschman, A. O. (1958). The Strategy of Economic Development, New Haven, Yale University Press, $217 p$

Ickis, J. C. (1998). Cómo ganar ventaja competitiva en el desarrollo económico local, Guatemala. [En línea] Disponible en: http://www.yorku.ca/ishd/CUBA.LIBRO.06/DEL/CAPITULO13.pdf. Consultado 08-08-2012.

Semestre Económico, volumen 16, №. 33, pp. 127-154 • ISSN 0120-6346, enero-junio de 2013, Medellín, Colombia 
Isard, W. (1956). Location and space economy: a general theory relating to industrial location, market areas, land use trade and urban structure. MIT Press, 350 pp.

Langen, P. (2004). The performance of port clusters: an extension of the cluster concept and an application to the port cluster of Rotterdam, Durban and the Lower Mississippi. Rotterdam: Erasmus University, PhD Thesis.

Lastres, H. (2007). Avaliação das políticas de promoção de arranjos produtivos locais no Brasil e proposição de ações. RedeSist, Rio de Janeiro, 37 pp.

Lastres, H. M. y Cassiolato, J. E. (2006). Innovación, información y conocimientos: la importancia de distinguir el modo de la moda, pp. 101 117. En: Gallina, A; Nuñez Jover, J; Capecchi, V; Arriete, L. Innovaciones creativas y desarrollo humano. Proyecto ALFA Lentisco. Ediciones Trilce. Itália, 320 pp.

Lastres, H.M. y Cassiolato, J.E. (2003). Novas políticas na era do conhecimento: o foco em Arranjos Produtivos e Inovativos Locais. RedeSist, Rio de Janeiro. En línea en http://www.redesist.ie.uf.

Lemos, C. (2003). Micro, pequenas e médias empresas no Brasil: novos requerimentos de polí ticas para a promoção de sistemas produtivos locais. Rio de Janeiro. Tese (Doutorado em Ciências) -COPPE- Universidade Federal, Rio de Janeiro.

Lewis, A. (1955). The theory of economicgrowth, Allen and Unwin, Londres, 453p.

Lopera, L. y Posada, G. (2009). Contribuciones de la economía solidaria al desarrollo local: El caso del altiplano norte del departamento de Antioquia. En: Semestre Económico, Vol. 12, N. ${ }^{\circ} 23$, pp. 119-132.

Lucas, R. (1988). On the mechanics of economic development. En: Journal of Monetary Economics, Vol. 22, N. ${ }^{\circ}$, pp. 3-42.

Lundvall, B.A. (1995). National Systems of Innovation: Towards a theory of innovation and interactive learning. Pinter, London, 342 pp.

Madoery, O. (2005). Políticas locales de desarrollo en Argentina. En: Revista Política y Gestión, Universidad Nacional de San Martín, Ed. Homo Sapiens, Buenos Aires, 30 pp.

Maillat, D. And Lecoq, B. (1992). New Technologies and the transformation of regional structures in Europe: the role of the milieu. En: Entrepreneurship and Regional Development, Vol. 4, pp. $1-20$.

Marshall, A. (1975). Princípios de economia política. São Paulo: Abril.

Marshall, A. (1890). Principles of Economics, Eighth Edition. London, Macmillan, 802 pp.

Maskell, P. y Malmberg, A. (1995). Localised learning and industrial competitiveness. En: Cambridge Journal of Economics, Vol. 23, N.ㄹ 2, pp. 167-185.

Méndez Gutiérrez Del Valle. (2006). Difusión de innovaciones en sistemas productivos locales y desarrollo territorial. Ponencia presentada II Congreso Internacional de la Red SIAL, España, 29 pp.

MDICEB -Ministério Do Desenvolvimento, Indústria E Comércio Exterior do Brasil- (2004). Termo de Referência para Política Nacional de Apoio ao Desenvolvimento de Arranjos Produtivos 
Acuerdos productivos desde la visión del desarrollo local: el rol de la innovación

Locais. [En línea]. Disponible en: http://www.desenvolvimento.gov.br/portalmdic/arquivos/ dwnl_ 1234181254.pdf. Consultado 05-08-2012.

Moré, J.D.; Lima, F. y Nascimento De Almeida. (2010). Modelo de gestión de um plan de desarrollo productivo local: El caso de Nova Friburgo, Brasil. En: Journal of Technology Management Innovation, Vol. 5, N. ${ }^{\circ}$ 3, pp. 192-205.

Morgan, K. (1995). The learning region: institutions, innovation and regional renewal. En:Regional Studies, Vol 31, N. ${ }^{\circ}$, pp. 491-503.

Myrdal, G. (1957). Teoría económica y regiones subdesarrolladas, Fondo de Cultura Económica, México, 188 pp.

Narayan, D. y Pritchett, L. (1997). Cents and Sociability: Household Income and Social Capital in Rural Tanzania. World Bank Research Working Paper, No 1796, Washington, D.C., Banco Mundial, $42 \mathrm{pp}$.

Narváez, M., Fernández, G., Senior, A. (2008). El desarrollo local sobre la base de la asociatividad empresarial: una propuesta estratégica. En: Opción, Vol. 24, N. 57, pp. 74-92.

Nelson, R. (1993). National Innovation Systems: a comparative study. Oxford University Press Oxford, $560 \mathrm{pp}$.

Nelson, R. R. y Winter, S. G. (1982). An Evolutionary Theory of Economic Change. Harvard University Press, Cambridge (MA), $454 \mathrm{pp}$.

Nuñez Jover, J; Montalvo Arriete, L. y Pérez Ones, I. (2006). Universidad y desarrollo social basado em el conocimiento: nuevas estratégias desde lo local, p. 165-182. En: Gallina, A; Nuñez Jover, J; Capecchi, V; Arriete, L. Innovaciones creativas y desarrollo humano. Proyecto ALFA Lentisco. Ediciones Trilce. Italia, 320 pp.

Nurkse, R. (1952). Some international aspects of the problem of economic development. En: American Economic Review, Vol. 42, N. ${ }^{\circ}$ 2, pp. 571-583.

Pérez, B. y Carrillo, E. (2000). Desarrollo local: manual de uso. ESIC Editorial, 668 pp.

Perroux, F. (1967). A economia do século XX. HERDER. Lisboa, 758 pp.

Piore, M.J.; Sabel, C.F. (1984). The second industrial divide: possibilities for prosperity. New York, $372 \mathrm{pp}$.

PNUD(1998). Integrating human rights with sustainable human development: A PNUD policy document. New York, USA, 32 pp.

Porter, M. E. (1990). Vantagem Competitiva: criando e sustentando um desempenho superior, $23^{\mathrm{a}} \mathrm{ed}$. Rio de Janeiro: Campus, $831 \mathrm{pp}$.

Putnam, R. (1995). Bowling alone: America's declining social capital. En: Journal

of Democracy, Vol. 6, N. ${ }^{\circ}$ 1, pp. 18-33.

Reinert, E. y Reinert, S. (2003). Innovation system of the past: modern nations-states in a historical perspective. The role of innovations and systemic effects in economic thought and policy. En The First Globelics Conference, Rio de Janeiro. 
Richardson (1986) Economía regional y urbana. Alianza Editorial, 309 pp.

Romer, P. (1990). Endogenous technical change. En: Journal of Political Economy, Chicago, Illinois, The University of Chicago Press, S71-S102.

Rostow, W. W. (1960). The Stages of Economic Growth. A Non-Communist Manifesto. Cambridge university Press, 272 pp.

Sachs, I. (1974). Ecodevelopment. En: Ceres, Vol. 17, N. ${ }^{4}$, pp. 17-21

Schumpeter, J. A. (1934). The Theory of Economic Development, Cambridge, Harvard University Press, $255 \mathrm{pp}$.

Simón, H.A. (1955). Behavioral Model of Rational Choice. Cowles Fundation Paper 98. The Quarterly Journal of Economics, vol. LXIX.

Sousa Leite, T. (2004). Encontro produtivo e ancoragem territorial: coordenação e relações firma-território em arranjos produtivos agroindústrias. Tesis de doctorado, Instituto de ciências humanas e sócias, Universidade Federal Rural de Rio de Janeiro.

Storper, M. (1997). The Regional World: Territorial Development in a Global Economy. Guildford Press, $338 \mathrm{pp}$.

Street, C. y Cameron, A.F.( 2007). External relationships and the small business: A review of small business alliance and network research. En: Journal of Small Business Management, Vol. 45, N. ${ }^{\circ}$, pp. 239-266.

Suzigan, W.; Furtado, J.; Garcia, R. y Sampaio, S. E. K. (2003). Aglomerações industriais no Estado de São Paulo. Instituto de Economia. UNICAMP.

Texeira, F. Y Ferraro, C. (2009). Aglomeraciones productivas locales en Brasil, formación de recursos humanos y resultados de la experiencia CEPAL-SEBRAE. Santiago de Chile.

United Nations Industrial Development Organization (UNIDO) (2001). Development of clusters and networks of SMEs. The UNIDO/ FEDEREXPORT Program (A guide to export consortia). Vienna.

Visser, E. (2004). A chilean wine cluster? Governance and upgrading in the phase of internationalization. United Nations Publication, ECLAC/GTZ project on Natural Resource-based strategies development. Chile.

Vasconcellos, G.M.(2007). Territórios vitoriosos. O papel das redes organizacionais. Editora Garamond.

Vázquez Barquero, A. (1998). Desarrollo endógeno. Conceptualización de la dinámica de las economías urbanas y regionales. En: Cuadernos del Cendes, N. 38, pp. 45-65.

Williamson, 0. (1975). Markets and Hierarchies: Analysis and Antitrust Implications. Free Press, New York. 